\title{
High- Powered Microwaves Ablation: An Emerging and Safe Tool for Treatment of Intrahepatic Cholangiocarcinoma
}

\author{
Antonio Giorgio*1, Pietro Gatti ${ }^{2}$, Luca Montesarchio ${ }^{3}$ and Valentina Giorgio ${ }^{4}$ \\ ${ }^{1}$ Interventional Ultrasound Unit, Tortorella Clinical Hospital, Salerno, Italy \\ ${ }^{2}$ Internal Medicine Unit, Brindisi General Hospital, Brindisi, Italy \\ ${ }^{3}$ General Surgery Unit, Polla General Hospital, Salerno, Polla (SA), Italy \\ ${ }^{4}$ Fondazione Policlinico A.Gemelli IRCCS, Department of Woman and Child Health and Public Health ; Roma- Italy \\ *Corresponding author: Antonio Giorgio , Viale Colli Aminei, 491 ; 80131 Naples - Italy
}

\section{ARTICLE INFO}

Received: 㓞 February 11, 2019

Published: 㓞 February 27, 2019

Citation: Giorgio A, Gatti P, Montesarchio L, Giorgio V. High- Powered Microwaves Ablation: An Emerging and Safe Tool for Treatment of Intrahepatic Cholangiocarcinoma. Biomed J Sci \& Tech Res 15(2)-2019. BJSTR. MS.ID.002674.

\begin{abstract}
This study aimed to compare retrospectively survival of 41 patients with Intrahepatic Cholangiocarcinoma (ICC) treated with percutaneous Radiofrequency Ablation (RFA) or high-powered Microwaves Ablation (MWSA) under Ultrasound Guidance. Patients treated with MWSA showed a significant better overall survival compared to patients treated with RFA either for nodules $<=3 \mathrm{~cm}$ than $>3 \mathrm{~cm}$. These results show that MWSA is superior to RFA in treating single $<=3 \mathrm{~cm}$ ICC nodules.
\end{abstract}

Abbreviations: Intrahepatic Cholangiocarcinoma; Ablation; Radiofrequency Ablation; Microwaves Ablation

\section{Introduction}

Cholangiocarcinoma $(\mathrm{CH})$ is the second primary malignant tumor of the liver. Intrahepatic Cholangiocarcinoma (ICC) is the third form of $\mathrm{CH}$ in order of frequency [1]. The treatment of choice is resection [2]. Ablation is considered one of the alternative tools if resection is unsuitable [3]. Radiofrequency ablation (RFA) was the first procedure that was employed using heat [4]. Compared to RFA, recent new generation Microwaves ablation (MWSA) is becoming a valid technique in treatment of ICC due to its high capability in inducing larger volume of necrosis in a faster time and without the so called "heat sink effect" [5,6]. Furthermore, high powered MWSA allows an increased sphericity of the area of necrosis [7]. Due to the above-mentioned advantages a margin of $0.5-1 \mathrm{~cm}$ of necrotic tissue can be easily obtained beyond the tip of the antenna [3]. The aim of this short communication was to report our results in treating ICC with MWS ablation compared with RFA.

\section{Materials and Methods}

Results of this retrospective study were obtained on 41 ICC patients (age range 58- 83 years, mean 70; 23 males) with 48 nodules treated with RFA (16 patients [39\%]) or MWS (25 patients [61\%]) between january 2008 and june 2018 in two Ultrasound (US) interventional Units in Southern Italy. Diameter of the nodules ranged between 2.1 and $7.2 \mathrm{~cm}$ and 14 patients (34.1\%) have underlying liver cirrhosis. RFA or MWSA was performed after surgical resection in 8 patients (19.5\%) and as first tool in the remaining patients. All diagnoses were obtained with percutaneous US - guided biopsy of the nodules using a 18G cutting needle. All ablation procedures were performed percutaneously under US guidance, with patients under unconscious sedation. RFA was performed using cold needles. MWSA abaltion was performed 
with a single antenna operating at $2450 \mathrm{MHz}$ and high-powered generator working between 100 - 140 watts.

\section{Statistical Analysis}

Cumulative Overall survival (OS) was calculated with the Kaplan-Meier method, and statistical comparison of survival distribution was analyzed by the log-rank test.

\section{Results}

The follow up ranged between 6 and 81 months. All procedures were completed, and no major complications occurred. Patients were discharged from the hospital the day after procedure after clinical and US examination and the hospital stay ranged between 2-4 days (mean 2.4). Overall survival of all treated patients was $87 \%, 74 \%, 44 \%$ and $33 \%$ at $12,36,60$ and 80 months, respectively (Figure 1). Median OS was 60 months. Mean OS of MWS group was 62 months and mean OS of RF group was 46 months $(\mathrm{p}<0.05)$. There was a significant statistically difference $(\mathrm{p}<0.05)$ between OS of MWS ablated patients $(77.9 \%, 65 \%$ and $65 \%$ at 36, 69 and 80 months, respectively) compared with RFA group (68.8 \%, 31.3 $\%$ and $18 \%$ at 36, 60 and 80 months, respectively) (Figure 2). Furthemore, patients with ICC nodules lesser than or equal to 3 cm treated with MWS survived longer (100\%, 80\%, 80\% and $80 \%$ at 12, 36, 60 and 75 months, respectively) than patients treated with RF $(100 \%, 100 \%, 42 \%$ and $28 \%$ at $12,36,60$ and 80 months, respectively) $(\mathrm{p}<0.05)$ (Figure 3). This was also more evident when we compared OS of MWS treated patients with OS of RF treated patients with ICC nodules larger than $3 \mathrm{~cm}$ (Figure 4).

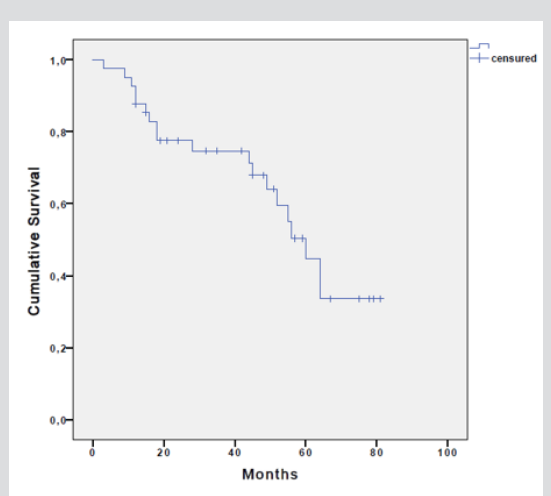

Figure 1: Overall survival of patients of our series.

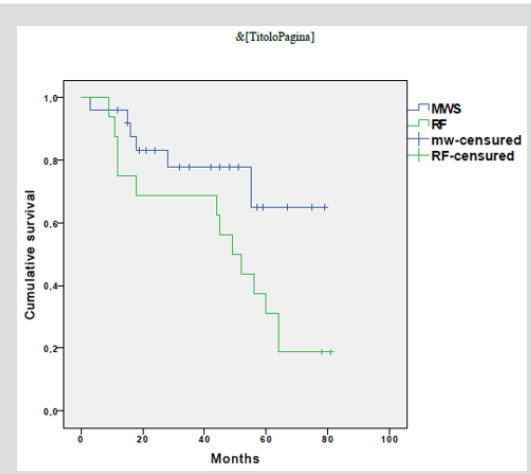

Figure 2: Overall survival of patients treated with MWSA (blue line) and RFA (green line).

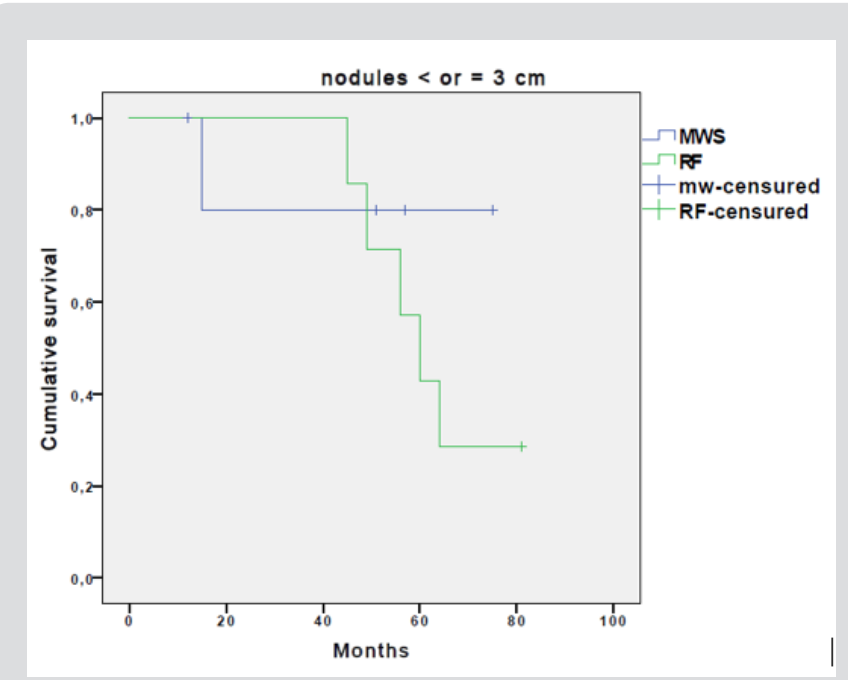

Figure 3: Overall survival of patients with ICC nodules <= $3 \mathrm{~cm}$ treated with MWSA (blue line) and RFA (green line).

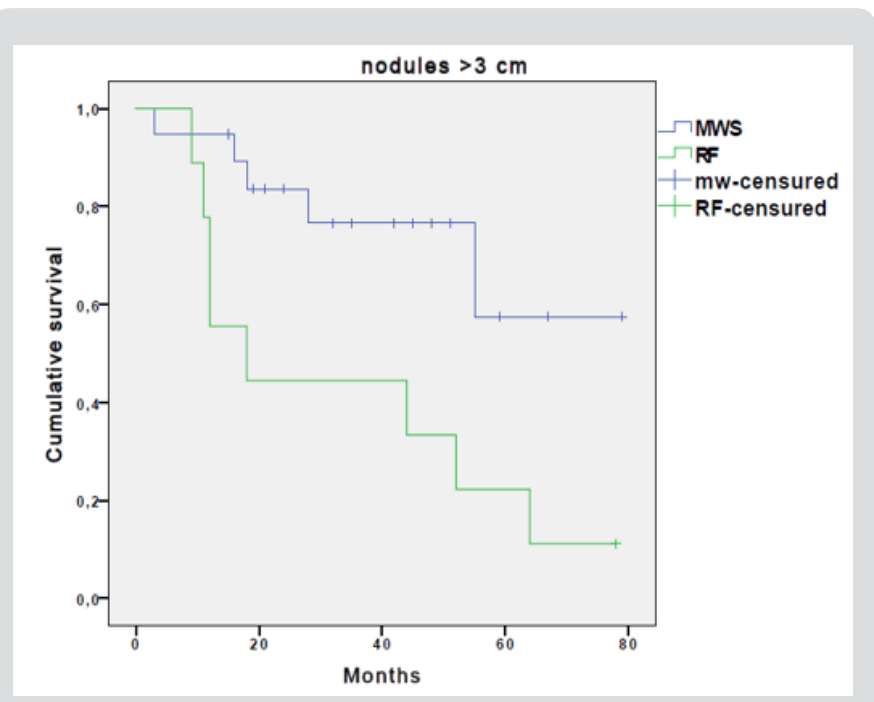

Figure 4: Overall survival of patients with ICC nodules $>3$ $\mathrm{cm}$ treated with MWSA (blue line) and RFA (green line).

\section{Discussion}

Minimally invasive ablative therapies have gained great popularity in treating primary liver tumors, mainly Hepatocellular carcinoma (HCC) in cirrhosis [8]. Based on good results obtained in treatment of HCC using heat, several authors performed thermal ablation in ICC patients unsuitable for resection [9-11]. The first thermal technique used for ablation was radiofrequency (RF). But, with the introduction of new generation and more powered MWS, a larger number of patients were treated using this new techique. Considering the advantages of high powered MWS over RF (larger volume of necrosis, sphericity of ablated lesions and especially easy capability to obtain at least $5 \mathrm{~mm}$ of ablation margin [3]), it is conceivable that MWSA should obtain increased benefit in terms of survival when ICC patients unsuitable for resection are treated with MWSA. Our results, although retrospective, seem to go in this direction. In our experience ICC patients treated with MWSA survived longer than patients treated with RFA. Furthermore, it is 
worth of mention that increased survival was observed either in MWS ablated patients with $<=3 \mathrm{~cm}$ nodules but also in MWS ablated patients with ICC nodules larger than $3 \mathrm{~cm}$. This would indicate that these results should be due to a technique capable to achieve in few minutes large volume of necrosis up to $4-6 \mathrm{~cm}$ [3]. In our study most of patients have a single nodule and therefore it is conceivable that a better local control of the tumor due to MWS characteristics can induce longer survival. Therefore, according to our results, single, small $<=3 \mathrm{~cm}$ ICC nodules, not suitable for surgery, should be treated no longer with RFA but rather with MWSA, exactly as it happens for small HCC in cirrhosis. Obviously, our study has many limitations. The first is that it is a retrospective study and, mainly, that our results were obtained in a not controlled manner. Therefore, further prospective, controlled, randomized studies, on a larger number of patients are needed.

\section{References}

1. Gupta A, Dixon E (2017) Epidemiology and risk factors: intrahepatic cholangiocarcinoma. Hepatobiliary Surg Nutr 6(2): 101-104.

2. Weber SM, Ribero D, O’Reilly EM, Kokudo N, Miyazaki M, et al. (2015) Intrahepatic cholangiocarcinoma: expert consensus statement. HPB (Oxford) 17(8): 669-680.

3. Giorgio A, Gatti P, Matteucci P, Giorgio V (2018) Ablative therapies for intrahepatic cholangiocarcinoma) Hepatobiliary Surg Nutr 7 (3): 192 194.

\section{ISSN: 2574-1241}

DOI: 10.26717/BJSTR.2019.15.002674

Antonio Giorgio. Biomed J Sci \& Tech Res

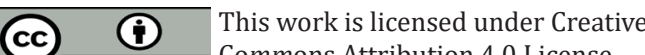

Submission Link: https://biomedres.us/submit-manuscript.php
4. Rossi S, Di Stasi M, Buscarini E, Quaretti P, Garbagnati F, et al. (1996) Percutaneous RF interstitial thermal ablation in the treatment of hepatic cancer. AJR AmJRoentgenol 167(3): 759-68.

5. Vogl TJ, Nour-Eldin NA, Hammerstein RM, Panahi B, Naguib NNN (2017) Microwave Ablation (MWA): Basics, Technique and Results in Primary and Metastatic Liver Neoplasms Rofo. 189(11): 1055-1066.

6. Giorgio A, Gatti P, Montesarchio L, Merola MG, Amendola F, et al. (2018) Microwave Ablation in Intermediate Hepatocellular Carcinoma in Cirrhosis: An Italian Multicenter Prospective Study. J Clin Transl Hepatol 6(3): 251-257.

7. Poggi G, Montagna B, DI Cesare P, Riva G, Bernardo G, et al. (2013) Microwave ablation of hepatocellular carcinoma using a new percutaneous device: preliminary results. Anticancer Res 33(3): 12211227.

8. Tan W, Deng Q, Lin S, Wang Y, Xu G (2019) Comparison of microwave ablation and radiofrequency ablation for hepatocellular carcinoma: a systematic review and meta-analysis. Int J Hyperthermia 24: 1-9.

9. Chiou YY, Hwang JI, Chou YH, Wang HK, Chiang JH, et al. (2005) Percutaneous ultrasound-guided radiofrequency ablation of intrahepatic cholangiocarcinoma. Kaohsiung J Med Sci 21(7): 304-309.

10. Fu Y, Yang W, Wu W, Yan K, Xing BC, et al. (2012) Radiofrequency ablation in the management of unresectable intrahepatic cholangiocarcinoma. J Vasc Interv Radiol 23(5): 642-649.

11. Giorgio A, Calisti G, DE Stefano G, Farella N, DI Sarno A, et al. (2011) Radiofrequency ablation for intrahepatic cholangiocarcinoma: retrospective analysis of a single centre experience. Anticancer Res 31(12): 4575-4580.

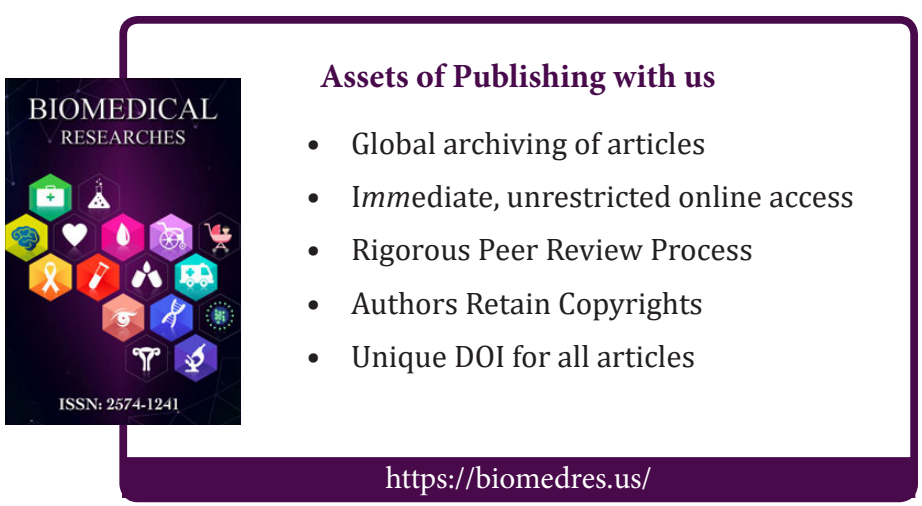

